\title{
Oncolytic Virus Therapy
}

National Cancer Institute

\section{Source}

National Cancer Institute. Oncolytic Virus Therapy. NCI Thesaurus. Code C62713.

A targ eted therapy that uses viral infection to cause the lysis of tumor cells. 